\title{
Histopathological diagnosis of small melanocytic lesions suspicious for malignant melanoma*
}

\author{
Danielle Carvalho Quintella ${ }^{1}$ \\ Leonardo Pereira Quintella ${ }^{3}$
}

\author{
Gabriella Campos-do-Carmo² \\ Tullia Cuzzi ${ }^{1}$
}

DOI: http:/ / dx.doi.org/10.1590/abd1806-4841.20175169

\begin{abstract}
The concern about malignant skin neoplasms leads to the excision of smaller lesions. This study on small melanocytic lesions aims to evaluate the range of possible histopathological diagnoses, describe histopathological aspects, and assess the usefulness of serial histological sections. We performed a cross-sectional descriptive histopathological study examining 76 pigmented skin lesions up to $6 \mathrm{~mm}$ in diameter. Histopathological diagnoses included atypical melanocytic nevi ( $\mathrm{n}=38$ ), common melanocytic nevi $(\mathrm{n}=18)$, atypical lentiginous melanocytic hyperplasia with architectural features of atypical melanocytic nevi $(n=7)$, lentigo simplex $(n=2)$, and malignant melanoma $(n=1)$. Ten cases were non-diagnostic. Cytological atypia was not an exclusive finding of atypical lesions. Examination of serial sections did not change histopathological impression. Early detection of malignant melanoma is important, but clinical and dermoscopy exams may be leading to the resection of a great number of benign lesions. Strict attention to histopathological criteria results in a large number of non-diagnostic cases. Keywords: Diagnosis; Melanoma; Nevi and melanomas; Pathology
\end{abstract}

\section{INTRODUCTION}

Early detection of malignant melanoma (MM) is critical for a good prognosis and its eventual cure. Combined with clinical examination, dermoscopy helps to determine whether pigmented skin lesions are melanocytic and suspicious of malignancy and reduces the number of unnecessary biopsies. ${ }^{1,2}$ However, the diagnostic accuracy of dermoscopy significantly depends on the degree of experience of the examiners and size of lesions. ${ }^{3-5}$ Small melanocytic lesions remain a significant diagnostic challenge not only for clinicians and dermoscopists, but also for pathologists because progressive smaller lesions are excised under the screening of dermoscopic features. This study aims to evaluate the range of possible histopathological diagnoses in a group of melanocytic lesions up to 6 $\mathrm{mm}$ considered suspicious for MM on clinical and dermoscopical examination. We carefully applied histopathological parameters focusing on melanocytic dysplasia and MM trying to achieve clinical-pathological correlation.

\section{MATERIALS AND METHODS}

Inclusion criteria consisted of patients attending the Dermoscopy Outpatient Clinic of the National Cancer Institute (Rio de Janeiro, Brazil) examined by a dermatologist with experience in dermoscopy and referred to pigmented skin lesion excision due to MM suspicion. Dermoscopic criteria used to qualify lesions as suspicious were based on pattern analysis. ${ }^{6}$
Biopsy specimens were fixed in $10 \%$ buffered formalin. Lesions were measured in the fixed gross specimen. Gross handling of the specimen was made so that the largest diameter of the lesion was analyzed microscopically.

After histological processing and paraffin embedding, we obtained 3 sets of 4 microtome sections. Each section was $4 \mu \mathrm{m}$ thick. Each set of 4 sections was placed on a slide. The staggering was performed choosing three sections of $4 \mu \mathrm{m}$ from each set of four selected sections

Cases were analyzed simultaneously by an experienced dermatopathologist and a pathologist with five-years of experience in diagnostic pathology in order to establish the histopathological diagnosis of the excised lesions.

Minimum criteria for the histopathological diagnosis of different entities were based on widely accepted standard criteria. Criteria for atypia/dysplasia were adapted from Crowson. ${ }^{7}$ Criteria were minutely analyzed and 59 variables treated as dichotomous. Data collected were entered into EpiData 3.1 software. Univariate analysis (frequency) was performed using SPSS 16 software. Emphasis was given to the architectural pattern of the junctional proliferation, circumscription of the lesion, symmetry, cytological features of junctional melanocytes, acanthosis, and papillary dermis fibroplasia. 
We excluded lesions located at acral or other special sites, lesions larger than $6 \mathrm{~mm}$, incompletely excised lesions, or those with processing artifacts.

\section{RESULTS}

Ninety biopsies were performed. Five were from special sites, 5 had processing artifacts, 3 were larger than $6 \mathrm{~mm}$, and 1 was incompletely excised. After excluding these 14 samples, 76 lesions from 43 patients were analyzed.

Twenty-nine $(67.4 \%)$ patients were female with an age range from 9-84 years (mean age 45.1, median age 48). All patients had personal or family history of MM. Lesions were located on the back (43.4\%), upper and lower limbs (35.5\%), trunk (18.4\%), and head and neck (2.6\%).

\section{Histopathological analysis}

Histopathological diagnoses included atypical junctional nevus, atypical compound nevus, common compound nevus, intradermal nevus, atypical lentiginous melanocytic hyperplasia with architectural features of atypical nevus, lentigo simplex, and MM. Some cases were non-diagnostic. Frequencies and sizes are shown in table 1.

The minimum histological criteria for the diagnosis of atypical junctional nevus $(n=21)$ were atypical junctional melanocytic proliferation with nests, acanthosis, and stromal changes. An intradermal component was added to the diagnosis of atypical compound nevus $(n=17)$. All cases presented lentiginous junctional melanocytic proliferation associated with nests. Nevocellular nests were located at the base of the rete ridges, except in one case of atypical junctional nevus. Nests located at lateral borders of the rete ridges or with an additional suprapapillary location were noted in both atypical junctional and compound nevi. Lateral limits (at least one) of the junctional component represented by isolated cells were more frequently observed in atypical junctional nevi $(76.2 \%)$ than in atypical compound nevi (47.1\%). For compound lesions, the extension of the junctional component for three or more rete ridges beyond the intradermal component (shoulder sign) was noted in all cases of atypical compound nevus and was symmetrical in most of them $(64.7 \%)$.

Atypical junctional nevi $(\mathrm{n}=21)$, atypical compound nevi $(\mathrm{n}=17)$, and atypical lentiginous melanocytic hyperplasia with ar- chitectural features of atypical nevus $(n=7)$ showed cytological atypia of junctional melanocytes, which was observed as increased nuclear size (compared to basal keratinocytes), changes in nuclear shape, chromatin pattern, and cytoplasmic expansion. Nuclei of proliferating melanocytes were increased up to 1.5 times in all cases, except in the case diagnosed as MM, in which nuclei were two times greater. The finding of nuclei of intermediate size (between 1.5 and 2 times) was observed in some cases, focally or in a minority of cells. There was a predominance of stellate cells with scanty cytoplasm and ink spot chromatin. Epithelioid melanocytes (with clear and delicate chromatin and eventually a small nucleolus) were noted as part of melanocytic proliferation in most cases, particularly in compound lesions (52\%). An exclusive epithelioid morphology was observed in atypical compound nevi $(41.2 \%)$ and atypical junctional nevus (9.5\%). Epithelioid melanocytes were not seen in atypical lentiginous melanocytic hyperplasia with architectural features of atypical nevus.

Isolated suprabasal melanocytes were seen in 14/45 atypical lesions - namely atypical junctional nevi (6), atypical compound nevi (6), and atypical lentiginous melanocytic hyperplasia (2) - and were restricted to the central area of the lesion. The only case in which this finding was present in lateral margins was an atypical compound nevus.

Stromal changes taken as a minimum criterion for identification of atypical lesions included fibroplasia of the papillary dermis, proliferation of vessels, and the presence of inflammatory cells. Fibroplasia of the papillary dermis, especially the concentric eosinophilic type, was present in all cases except a case of atypical compound nevus.

Intradermal nevi $(\mathrm{n}=9)$ had a junctional lentiginous component in $77.8 \%$ of cases, with melanocytes increased nuclear size in $66.7 \%$. Shoulder sign was observed in $33.3 \%$ of cases and was asymmetrical in $11.1 \%$ of cases. Epidermal changes consistent with actinic keratosis were observed in 1 intradermal nevus.

Melanocytes with increased nuclear size (up to 1.5 times) were present in 2 cases diagnosed as lentigo simplex.

Malignant melanoma was represented by a symmetrical junctional melanocytic proliferation with isolated cells and nests (in situ) of epithelioid and stellate cells with nuclear size increased at

TABLE 1: Histopathological diagnosis and size of 76 melanocytic lesions suspicious for malignant melanoma

\begin{tabular}{|c|c|c|c|c|c|c|c|}
\hline \multirow[b]{2}{*}{ Histopathological diagnosis } & \multicolumn{6}{|c|}{ Size } & \multirow[b]{2}{*}{ Total n (\%) } \\
\hline & $1 \mathrm{~mm}$ & $2 \mathrm{~mm}$ & $3 \mathrm{~mm}$ & $4 \mathrm{~mm}$ & $5 \mathrm{~mm}$ & $6 \mathrm{~mm}$ & \\
\hline Atypical junctional nevus & - & 6 & 10 & 4 & 1 & - & $21(27.6)$ \\
\hline Atypical compound nevus & - & - & 6 & 5 & 4 & 2 & $17(22.4)$ \\
\hline Non-diagnostic & - & 5 & 3 & - & 2 & - & $10(13.2)$ \\
\hline Common compound nevus & - & 6 & - & 3 & - & - & $9(11.8)$ \\
\hline Intradermal nevus & 1 & 2 & 3 & 2 & 1 & - & $9(11.8)$ \\
\hline $\begin{array}{l}\text { Atypical lentiginous melanocytic } \\
\text { hyperplasia with architectural features } \\
\text { of atypical melanocytic nevus }\end{array}$ & 1 & 3 & - & 2 & 1 & - & $7(9.2)$ \\
\hline Lentigo simplex & - & 1 & - & - & 1 & - & $2(2.6)$ \\
\hline Malignant melanoma & - & - & 1 & - & - & - & $1(1.3)$ \\
\hline Total n (\%) & $2(2.6)$ & $23(30.2)$ & $23(30.2)$ & $16(21.1)$ & $10(13,2)$ & $2(2.6)$ & $76(100)$ \\
\hline
\end{tabular}


least 2 times (Figure 1). Suprabasal melanocytes were present in the central area of the lesion, epidermis showed mild acanthosis, and there was focal lamellar fibroplasia in the papillary dermis.

Ten cases were non-diagnostic because they did not meet the minimum histological criteria of classically established entities. All of them were represented by a lentiginous junctional melanocytic proliferation with epithelioid morphology and increased nuclear size in the acanthotic epidermis. Eight of these cases had $3 \mathrm{~mm}$ or less. Nests were seen in 2 lesions. Suprabasal dissemination of melanocytes (restricted to the central area of the lesion) was seen in 8 cases. Fibroplasia of the papillary dermis was identified in 4 . In 1 case, junctional melanocytes exhibited an expanded eosinophilic cytoplasm (spitzoid morphology) without nest formation together with a few isolated intradermal cells (Figure 2).

\section{DISCUSSION}

This study was based on a comprehensive histopathological analysis of a convenience sample composed of 76 sequentially enrolled melanocytic lesions with $6 \mathrm{~mm}$ or less in diameter. Patients were followed at the Dermoscopy Outpatient Clinic, which justifies the fact that we found only 3 cases larger than $6 \mathrm{~mm}$. Dealing with prospective collected samples and clinical/dermoscopic analyses,

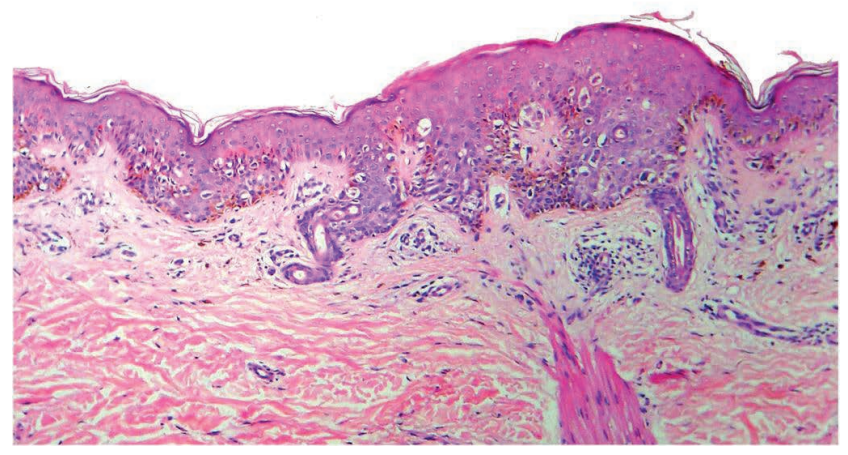

Figure 1: Malignant melanoma in situ. Junctional melanocytic proliferation with severe cytological atypia and focal pagetoid dissemination. Acanthosis, pigmentation of basal queratinocytes, and inflammatory infiltrate around ectatic vessels associated(Hematoxylin \& eosin $x 100)$

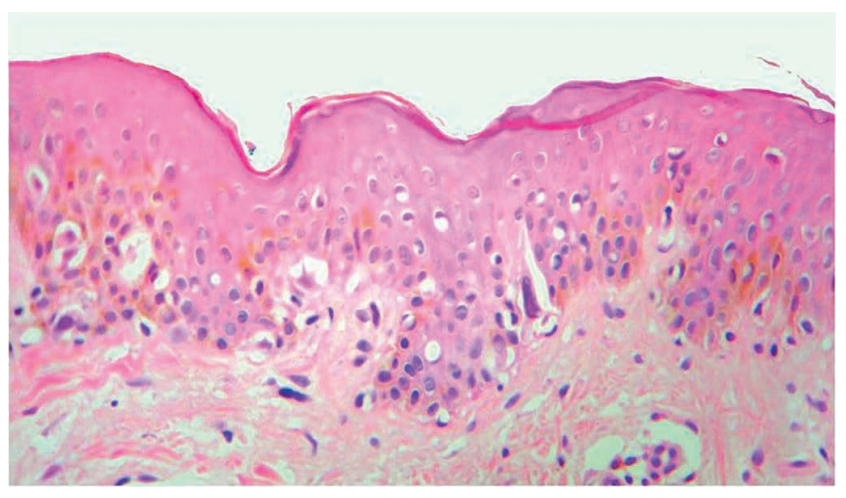

FIGURE 2: Non-diagnostic case. Junctional melanocytic proliferation with severe cytological atypia and spitzoid features in an 18-year-old boy (Hematoxylin \& eosin x400) we were able to expand the histopathological spectrum of diagnosis as compared to other previous reports. ${ }^{8}$ Although serial sections were done, which improves the exam of lesions, we did not find that they modify the initial impression regarding biological behavior.

Morphological criteria used to diagnose atypical larger lesions allowed diagnosis of atypical lesions up to $6 \mathrm{~mm}$, but these criteria should be carefully weighed and combined. Although cytological melanocytic atypia is one of two major criteria necessary for the diagnosis of atypical melanocytic nevi, it is not an exclusive finding of atypical lesions and, in our study, this criterion was present in common melanocytic nevi, as previously reported. ${ }^{7,9}$ Moreover, in one specific case of compound melanocytic nevus, cytological criteria and stromal changes required for the diagnosis of atypical compound melanocytic nevus were seen, including lamellar fibroplasia. We preferred to classify this case as a common melanocytic nevus because of its symmetry and circumscription and because the criteria for dysplasia were focal - although our analysis did not take quantitative aspects into account (data not shown). This lesion had 2 $\mathrm{mm}$, and criteria for dysplasia encountered were more likely related with its morphological immaturity. Possibly, this compound melanocytic lesion could be undoubtedly classified as typical or atypical in a later stage of development.

According to Carli and colleagues, dermoscopy is less useful for the diagnosis of lesions up to $6 \mathrm{~mm}$ in diameter than in larger lesions. ${ }^{5}$ This seems to be in agreement with the present study, in which only 1 out of 76 suspicious lesions was diagnosed as malignant. Ordinary benign melanocytic lesions usually are not clinically suspicious. Notwithstanding, all cases diagnosed as benign proliferations without histopathological signs of atypia showed some other histopathological findings that could explain and correlate with clinical suspicion. Most of the intradermal melanocytic nevi studied had an associated lentiginous melanocytic proliferation, basal hyperpigmentation, and acanthosis that could justify clinical atypia. In one case, there was association with actinic keratosis. One of the two cases of lentigo simplex showed focal melanosis, which also may introduce a point of concern in dermoscopical analysis.

The malignant melanoma case was a 3-mm symmetric lesion. Asymmetry is a criterion for such diagnoses (described in clinical and dermoscopic $\mathrm{ABCD}$ rules), but MM lesions smaller than 3 $\mathrm{mm}$ in diameter may be symmetrical or asymmetrical. ${ }^{10-12}$ We should also remember that small lesions may not develop the spectrum of clinical, dermoscopical, and histopathological characteristics. Hence, careful surveillance, especially in risk groups, is justified. On the other hand, considering the increasing incidence of MM and its relatively stable mortality, the issue of overdiagnosis must be taken into account. ${ }^{13}$ As a result, although early detection of MM is important, we have to keep in mind that clinical and dermoscopical examinations can be leading to the resection of not only a great number of lesions that are not MM, but also of MM that would not cause symptoms or death. ${ }^{13}$

Strict attention to histopathological criteria resulted in $10 / 76$ cases without a conclusive diagnosis. We tended to classify these cases as de novo intraepidermal epithelioid melanocytic dysplasia, a recently described entity that is considered an MM by some authors. ${ }^{14,15}$ However, all of our cases showed acanthosis (at least fo- 
cal) or acanthosis associated with stromal fibroplasia, features that were not described in that entity. ${ }^{9,10}$ Atypical lentiginous melanocytic hyperplasia with architectural features of atypical nevus was also considered, but epithelioid morphology of melanocytes does not suit this diagnosis. Malignant melanoma could not be completely excluded, yet it did not seem appropriate because of the absence of continuous junctional melanocytic proliferation. One of the non-diagnostic cases was singular. It refers to a $3 \mathrm{~mm}$ lesion in a young patient (18 years old), represented by junctional lentiginous epithelioid melanocytic proliferation with spitzoid features, sometimes with elongated nuclei, enlarged, and prominent lavender-colored nucleoli. We were prompt to the diagnosis of pagetoid spitz nevus. However, besides acanthosis, a dermal component composed of a few isolated melanocytes was present. Keeping strict morphologic criteria, this case could not be diagnosed as a composed melanocytic

\section{REFERENCES}

1. Tran KT, Wright NA, Cockerell CJ. Biopsy of the pigmented lesion--when and how. J Am Acad Dermatol. 2008;59:852-71.

2. Cohen DE, Sangueza OP, Fass E, Stiller MJ. In vivo cutaneous surface microscopy: revised nomenclature. Int J Dermatol. 1993;32:257-8.

3. Bafounta ML, Beauchet A, Aegerter P, Saiag P. Is dermoscopy (epiluminescence microscopy) useful for the diagnosis of melanoma? Results of a meta-analysis using techniques adapted to the evaluation of diagnostic tests. Arch Dermatol. 2001;137:1343-50.

4. Kittler H, Pehamberger H, Wolff K, Binder M. Diagnostic accuracy of dermoscopy. Lancet Oncol. 2002;3:159-65.

5. Carli P, De Giorgi V, Chiarugi A, Nardini P, Mannone F, Stante M, et al. Effect of lesion size on the diagnostic performance of dermoscopy in melanoma detection. Dermatology. 2003;206:292-6

6. Argenziano G, Soyer HP, Chimenti S, Talamini R, Corona R, Sera F, et al. Dermoscopy of pigmented skin lesions: results of a consensus meeting via the Internet. J Am Acad Dermatol. 2003;48:679-93.

7. Crowson AN, Magro CM, Martin C. Mihm J. Dysplastic melanocytic nevi, de novo intraepidermal epithelioid and lentiginous melanocytic dysplasias, and nevi at specific anatomic sites. In: Crowson AN, Magro CM, Martin C. Mihm J, editors. The melanocytic proliferations: a comprehensive textbook of pigmented lesions. 2nd ed. Hoboken, NJ: Wiley-Blackwell 2014. p.201-53.

8. Braun-Falco M, Hein R, Ring J, McNutt NS. Histopathological characteristics of small diameter melanocytic naevi. J Clin Pathol. 2003;56:459-64.

9. Cook MG. Benign melanocytic lesions mimicking melanomas. Pathology. 2004;36:414-8.

10. Hazen BP, Bhatia AC, Zaim T, Brodell RT. The clinical diagnosis of early malignant melanoma: expansion of the $A B C D$ criteria to improve diagnostic sensitivity. Dermatol Online J. 1999:5:3.

11. Stolz W, Semmelmayer U, Johow K, Burgdorf WH. Principles of dermatoscopy of pigmented skin lesions. Semin Cutan Med Surg. 2003:22:9-20.

12. Bono A, Bartoli C, Baldi M, Moglia D, Tomatis S, Tragni G, et al. Micro-melanoma detection. A clinical study on 22 cases of melanoma with a diameter equal to or less than 3mm. Tumori. 2004;90:128-31. nevus because of the absence of nests at the dermo-epidermal junction; and could not be classified as lentigo simplex because of the presence of a dermal component, which, together with the presence of epithelioid cells, also invalidate the diagnosis of atypical lentiginous melanocytic hyperplasia with architectural features of atypical nevus. The diagnosis of de novo intraepidermal epithelioid melanocytic dysplasia again would not be suitable because of the presence of acanthosis, as well as lamellar fibroplasia. Finally, the melanocytic proliferation was not continuous, due to the diagnosis of MM. Most likely, again, we examined a not fully developed lesion, lacking enough histopathological characteristics for a definitive diagnosis to be made. This kind of restriction should be considered by clinicians and pathologists while dealing with such small lesions.]
13. Welch HG, Black WC. Overdiagnosis in cancer. J Natl Cancer Inst. 2010;102:605-13.

14. Sachdeva M, Frambach GE, Crowson AN, Deng AC, Mihm MC Jr, Magro CM. De novo intraepidermal epithelioid melanocytic dysplasia as a marker of the atypical mole phenotype -- a clinical and pathological study of 75 patients. J Cutan Pathol. 2005;32:622-8

15. Jessup CJ, Cohen LM. De novo intraepidermal epithelioid melanocytic dysplasia: a review of 263 cases. J Cutan Pathol. 2010;37:852-9.

How to cite this article: Quintella DC, Campos-do-Carmo G, Quintella LP, Cuzzi T. Histopathological diagnosis of small melanocytic lesions suspicious for malignant melanoma. An Bras Dermatol. 2017;92(3):375-8. 